\title{
Designing an Algorithm for Bioloid Humanoid Navigating in its Indoor Environment
}

\author{
Md. Akhtaruzzaman", Amir A. Shafie, Mahbubur Rashid \\ Department of Mechatronics Engineering, Kulliyyah of Engineering, International Islamic University Malaysia, Kuala Lumpur, 53100, \\ Malaysia
}

\begin{abstract}
Gait analyses are the preliminary requirements to establish a navigation system of a humanoid robot. Designing a suitable indoor environment and its mapping are also important for the android localization, selection of a goal to achieve it and to perform the assigned tasks in its surroundings. This paper delineates the various gaits like walking, turning, obstacle overcoming and step up-down stairs for a humanoid system. The writing also explicates the design of the indoor test environment with the stationary obstacles placed on the navigation routes. The development of an efficient algorithm is also excogitated based on the various analyses of gaits and the predefined map of the test environment. As the navigation map is predetermined, the designed algorithm animates the humanoid to navigate by selecting an optimal route, depending on some external commands, to reach at the goal position. Finally the performance of the system is analysed based on the elapsed time of the navigation action with the validation of optimal navigation strategy where the designed algorithm demonstrates the robustness of its implementation and execution.
\end{abstract}

Keywords Anthropomorphic Robot Navigation, Navigation Algorithm, Humanoid Robot, Optimal Navigation System

\section{Instauration}

A biped humanoid is considered as an open kinematic chain consisting of two sub chains as legs and torso which connected at a common point, the hip. Walking gait is defined as the alternating phases of single and double support mode. If only one leg is in contact with the ground is called as the stance leg while the other one is called as the swing leg. A planar biped is a biped with motions taking place only in the sagittal plane while the three dimensional walker has motions taking place in both the sagittal and frontal planes[1,2]. Reality is that, research in this area gathers multiple disciplines that introduce one of the challenging orbits in the Mechatronics Engineering.

In order to give an overview of navigation techniques, these can be classified based on indoor and outdoor navigation system. For indoor navigation system the easiest way of making a robot goes to a goal location is simply to guide to the location. This guidance can be done in different ways like burying magnets or inductive loops in the floor, painting lines on the floor by placing beacons, markers, barcodes etc. These techniques are commonly used in industrial scenarios for transportation tasks[1,4]. Motion planning tasks are the most important functions for a Humanoid Robot

* Corresponding author:

akhter900@yahoo.com (Md. Akhtaruzzaman)

Published online at http://journal.sapub.org/jmea

Copyright (C) 2012 Scientific \& Academic Publishing. All Rights Reserved
Navigation system. Motion planning algorithms might address the robots with a larger number of joints, more complex tasks, different constraints and uncertainty[5-8]. A basic problem of motion planning technique is to produce a continuous motion that connects the start configuration, $\mathrm{S}$, and a destination configuration, $\mathrm{D}$, while avoiding collision with known obstacles[10,11].

This paper demonstrates an experiment on Optimal Navigation System (ONS) for biped robot in its indoor environment with the development and implementation of a navigation algorithm. Section 2 represents the various gait analyses by focusing on the forward walking. Strategy in designing the various subroutines for walking, turning, stepping up-down stairs and obstacle overcoming gaits are also presented in the same section in a succinct manner. Section 3 draws a panoptic view on Indoor Navigation Map (INP) pattern. Design scheme of the main program structure to control the defined subroutines is illustrated in the section 4. In section 5, observations and result analysis of the overall system performance are presented with the validation of the optimal navigation system. Finally the paper draws its abridgement with the outcomes of the research.

\section{Analysis and Formulation of Gaits}

The gaits are the combination of several postures of the humanoid system which are characterized as poses. Some series of poses are grouped together to make a page and a group of pages are combined with a correct order to make the 
system able to grab the next stage of the corresponding gait. If the system starts to execute a particular page, the execution should be completed before the start of the next execution process. As the mathematical expression the execution

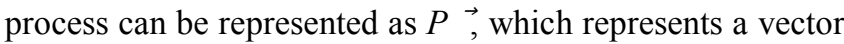
and a function of the joint vector $Q \overrightarrow{\text { }}$. The $P \overrightarrow{\text { indicates the }}$ poses of the humanoid system for a certain moment of time in a particular direction while executing the page. If the start-pose and the final-pose of a single page are indicated by $P_{i}$ and $P_{f}$, a general equation can be formulated as,

$$
\begin{gathered}
\vec{P}=\overrightarrow{P_{i} P_{f}}=\sum_{i}^{f} f\left(\vec{Q}_{(i)}\right) \\
\vec{Q}_{(i)}=\left\{\vec{Q}_{1(i)}, \vec{Q}_{2(i)}, \vec{Q}_{3(i)} \ldots \ldots \ldots, \vec{Q}_{n(i)}\right\}
\end{gathered}
$$

Here $Q_{(i)} \rightarrow$ is the set of joint vectors of ' $i$ ' th pose depending on the ' $n$ ' Degree of Freedom (DoF) of the system.

Humanoid walking is the combination of the continuous step actions attempted by the left or right leg. The walking gait is constructed depending on the three different steps which are accumulated together following a certain type of rules. The three types of steps are excogitated as, a) Initial Step (IS), b) Full Step (FS) and c) Ending Step (ES).

\subsection{Designing the Walking Initial Step (IS)}

The Initial Step (IS) has two types upon the foot in action to make the step, a) IS by left foot and b) IS by right foot. Both of these types are split into five poses.

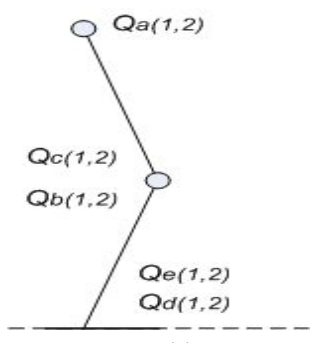

(a)

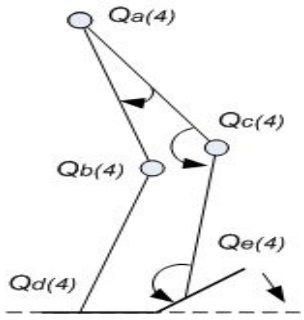

(c)

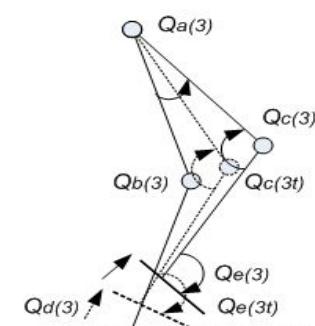

(b)

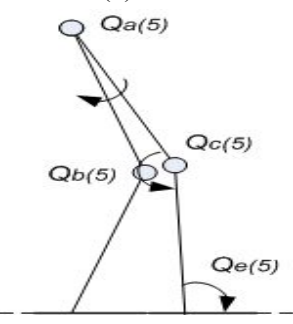

(d)
Figure 1. Five poses for Initial Step (IS), (a) Action and Tilt Poses (two poses), (b) Double support to Single support transition (DS-SS), (c) Single support to Double support transition (SS-DS) and (d) IS complete Pose

As the simplification of the system the lower torso is considered having five joint actuators as shown in Figure 1. The first two poses, Action Pose and Tilt Pose are represented in Figure 1 (a). The Tilt Pose represents the action while the system tilts to one side to place the position of the CoM point just directly above the supporting CoP point while the other foot is on swing. The DS-SS Pose is the starting of the swing action of the free leg to have the initial step. This pose has one transition stage as indicating by $Q_{e(3 t)}$ in Figure 1 (b) where the free foot moves up the hill while touching the tip with the surface and after a certain time, the foot becomes fully free from the ground achieving the new position indicating as $Q_{e(3)}$ shown in the same figure. In this position the Humanoid is on Single Support mode. This pose ends with the touching of the navigation surface by the hill of the swing foot as shown in Figure 1 (c). With the last pose when both feet become flat with the ground, the initial step ended up placing the CoM point closer to the rear foot support point. If the start and final stage of these poses are indicated as $P_{i}$ and $P_{f}$, the whole process can be represented as $P_{I} \rightarrow$ according to Equation (1).

$$
\begin{gathered}
\vec{P}_{I}=\overrightarrow{P_{i} P_{f}}=\sum_{i=1}^{f=5} f\left(\vec{Q}_{(i)}\right) \\
=\sum_{i=1}^{f=5} f\left(\vec{Q}_{a(i)}, \vec{Q}_{b(i)}, \vec{Q}_{c(i)}, \vec{Q}_{d(i)}, \vec{Q}_{e(i)}\right)
\end{gathered}
$$

Here $P_{I} \rightarrow$ is the Initial Step vector as the function of the joint vectors, $Q_{(i)}$, indicating the certain pose of the humanoid where every joint vectors of the system are internally related to each other. Based on the relation of the poses, an algorithm can be written both for the IS by left and right foot with the description of two functions, WIS_Left ( ) and WIS_Right (), as shown in Figure 2 (a) and (b).

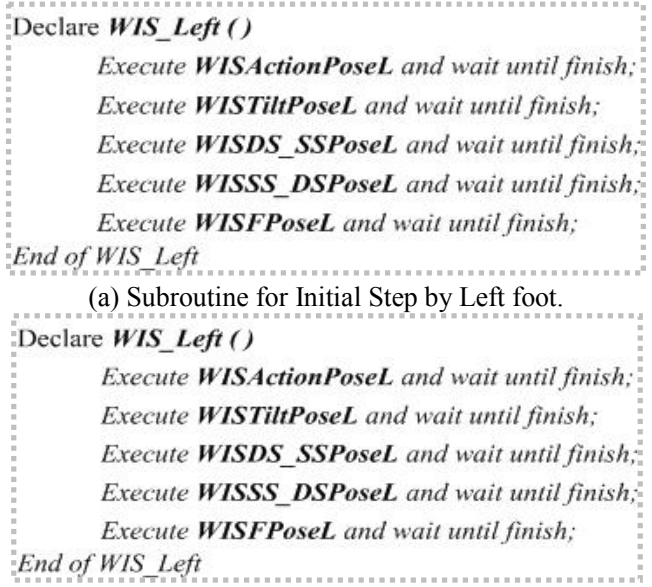

(b) Subroutine for Initial Step by Right foot.

Figure 2. Function definitions for Initial Step attempted by Left and Right foot

\subsection{Design of the Full Step (FS) and Ending Step (ES)}

Figure 3 represents the three functions to execute the three different steps of walking. The gaits for Full Step and Ending Step are designed by following the same strategy as shown in Figure 4 and Figure 5.

The algorithm for the FS and ES steps include four functions named as, WFS_Left(),WFS_Right(),WES_Left () and WES_Right (). The whole process of the two step patterns can also be represented using the same equation as described in Equation (1).

$$
\begin{gathered}
\vec{P}_{F}=\overrightarrow{P_{i} P_{f}}=\sum_{i=5}^{f=9} f\left(\vec{Q}_{(i)}\right) \\
=\sum_{i=5}^{f=9} f\left(\vec{Q}_{a(i)}, \vec{Q}_{b(i)}, \vec{Q}_{c(i)}, \vec{Q}_{d(i)}, \vec{Q}_{e(i)}\right) \\
\vec{P}_{E}=\overrightarrow{P_{i} P_{f}}=\sum_{i=9}^{f=13} f\left(\vec{Q}_{(i)}\right) \\
=\sum_{i=9}^{f=13} f\left(\vec{Q}_{a(i)}, \vec{Q}_{b(i)}, \vec{Q}_{c(i)}, \vec{Q}_{d(i)}, \vec{Q}_{e(i)}\right)
\end{gathered}
$$




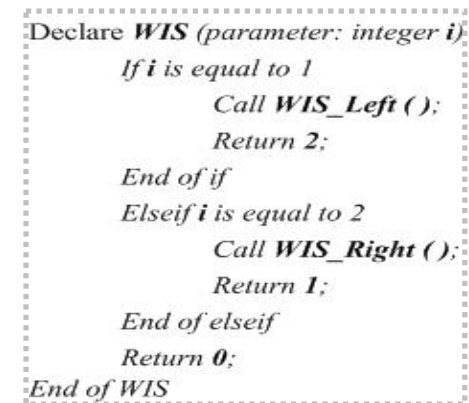

(a) Initial Step function.

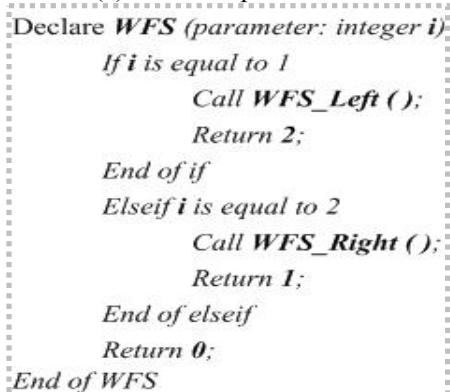

(b) Full Step function.

Declare $\boldsymbol{W E S}$ (parameter: integer $\boldsymbol{i}$ )

If $\boldsymbol{i}$ is equal to $I$

Call WES_Left ();

End of if

Elseif $i$ is equal to 2

Call WES_Right ();

\section{End of elseif}

Return 0;

End of WES

(c) Ending Step function.

Figure 3. Three different functions to execute the three different steps for walking

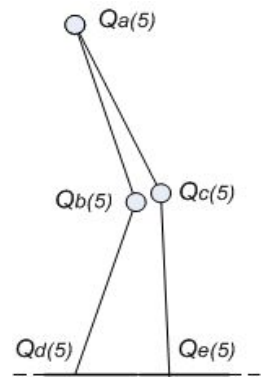

(a)

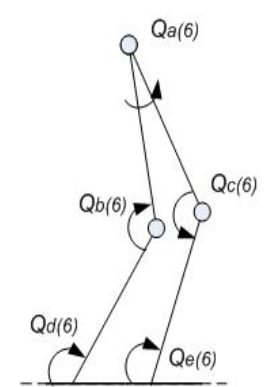

(b)

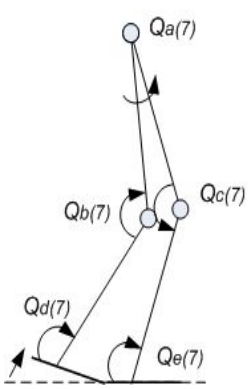

(c)

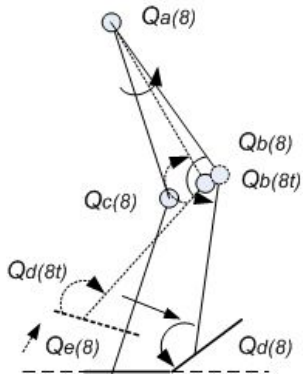

(d)

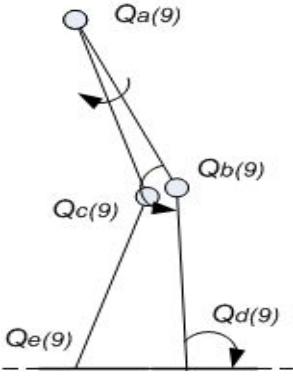

(e)
Figure 4. Five poses for Full Step (FS), (a) Action Pose, (b) Tilt Pose, (c) Double support to Single support transition (DS-SS), (d) Single support to Double support transition (SS-DS) and (e) FS Final Pose

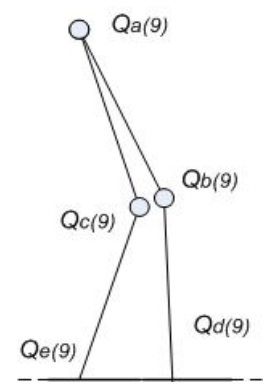

(a)

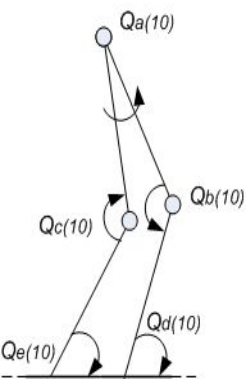

(b)

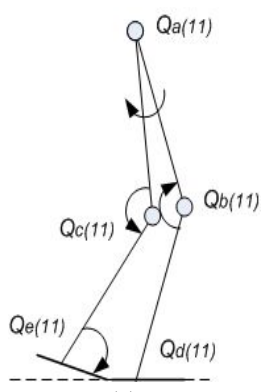

(c)

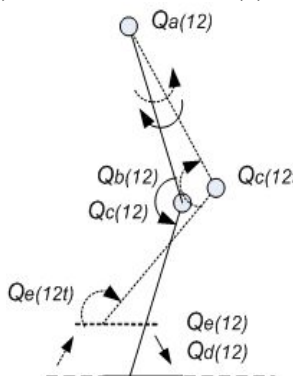

(d)

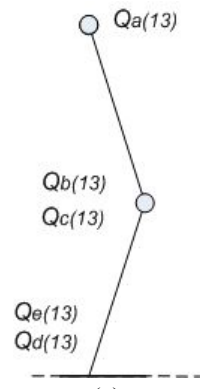

(e)
Figure 5. Poses for Ending Step (ES), (a) Initial Pose, (b) Tilt Pose, (c) Double support to Single support transition (DS-SS Pose), (d) Single support to Double support transition (SS-DS Pose) and (e) Action Pose

\subsection{Contriving the Various Subroutines}

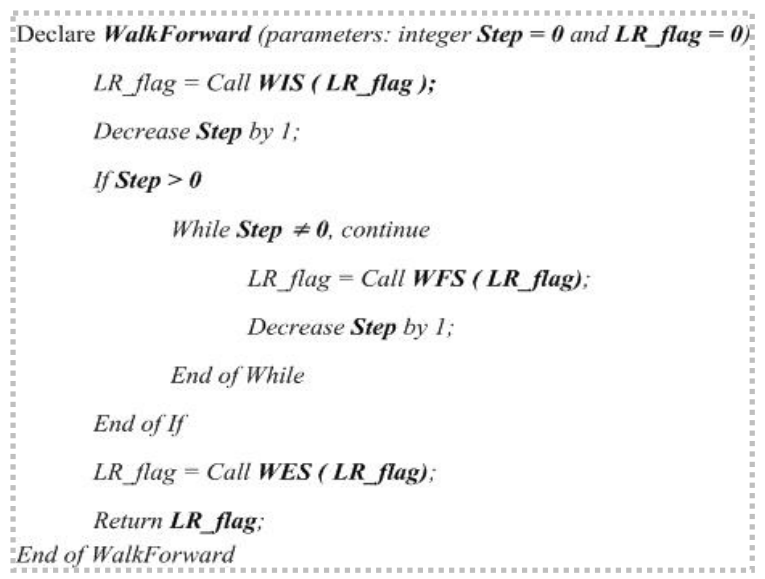

Figure 6. Main function to execute the continuous walking

For all the three functions, WIS(),WFS() and WES( ), a parameter ' $i$ ' is passed that indicates the action leg to generate the walking pattern. If the value of ' $i$ ' is 1 , the system will make a step by swinging its left leg. For the value of ' $i$ ' as 2 , the robot will perform its step by right leg. The return value, 1 or 2, indicates the action leg for the next step of the walking pattern, 1 is for Left and 2 is for Right. The return value ' 0 ' informs that the walking pattern just reached to the Final Pose of the Ending Step (ES) and the system is in the Action mode. The three different gaits are executed from a main function to perform the continuous walking which is defined by the function as WalkForward(), shown in Figure 6 , with two parameter values indicating the number of Steps and Left-Right indicator depending on the distance to reach the goal position.

To design the other necessary gaits for Obstacle Overcoming Step, Step Up-Down stairs and Turning left or right 
are also developed and the corresponding several algorithms are represented as, a) OOS_Left(), OOS_Right(), b) StUP_Left(), StUP_Right(), StDN_Left(), StDN_Right(), c) TuS_Left(), TuS_Right() respectively. The main functions for these several gaits are defined and declared as OOS(parameter: integer i), StUP(parameter: integer i), StDN(parameter: integer $i$ ) and TuS(parameter: integer $i)$.

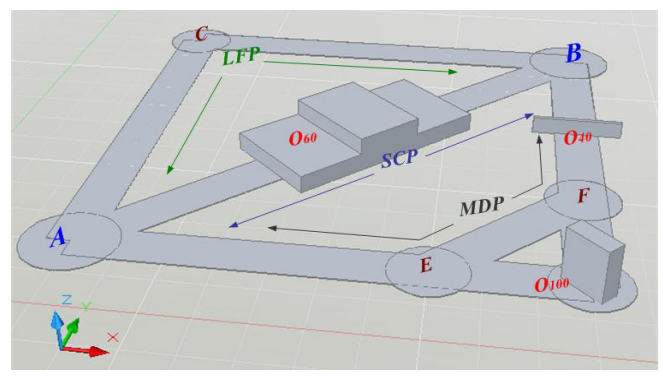

(a) Conceptual design of navigation map

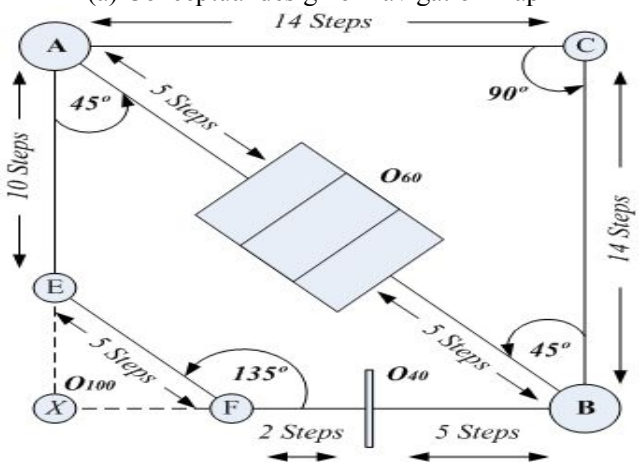

(b) Specification of the map

Figure 7. Conceptual design and specification of the indoor navigation map

\section{Environment Setup}

The navigation map is designed for the humanoid system where the robot is responsible to move from source position to destination point, $\mathrm{A}$ to $\mathrm{B}$ or $\mathrm{B}$ to $\mathrm{A}$ as shown in Figure 7 representing the conceptual model of the map. The source and the destination are connected with four different paths with various length and complexity. The direct path, $\mathrm{AB}$, from source to destination is considered as the shortest path where a complex obstacle, stairs indicated by $O_{60}$ in Figure 7 , is placed. The route is defined as Shortest Complex Path
(SCP). The longest track, ACB or BCA is the obstacle free path to move from origin to goal position and it is represented by the term, Longest Free Path (LFP). A lower high obstacle indicated by $O_{40}$ in Figure 7 is placed on the way of the medium path, AEFB or BFEA. The obstacle height is around $2.6 \mathrm{~cm}$ and the path is presented as Medium Difficult Path (MDP).

There exists another extra sub way, FOE, while travelling with the medium path. The sub way cannot be traced by the humanoid because of having an obstacle with the difficulty level as $100 \%$, which means the obstacle is unavoidable for the system. The different value of the obstacles are set as 100 , 60 and 40 as indicated by $O_{100}, O_{60}$ and $O_{40}$ on the navigation map, represents the various difficulty level in overcoming or avoiding them while travelling on the designed map. The map is predefined for the system and the obstacles are considered as stationary.

\section{Designing the Navigation Algorithm}

Since the humanoid has to travel through three different paths to navigate itself from source to destination, A to B or $\mathrm{B}$ to $\mathrm{A}$, it is necessary to define all the environment constraints so that the system can understand the map and localize itself while navigating. There could be various ways to define the navigation map such as Brute-Force Method, Structure Format, Tabular Format or Coordinate Frames. For this experiment, the Tabular Format is chosen as one of the best and easier techniques and is followed to design the navigation algorithm.

The robot moves by selecting the optimal path based on three different modes, Hurry, Medium and Relax. The three different modes are chosen depending on three different commands. If the system is in Hurry Mode, it will select the shortest path, SCP, to reach the goal within a short time although there exists a complex obstacle to come over. For the Medium Mode, the robot will walk on the MDP route having some turning movements with overcoming a difficult obstacle. In Relax Mode the humanoid will reach the goal position over the easiest path, LFP, where no obstacles are placed.

Table 1. Definition of the Map Constraints (Look up Table)

\begin{tabular}{|c|c|c|c|c|c|c|c|c|c|c|}
\hline Route & Target & \multicolumn{9}{|c|}{ Map Constraints } \\
\hline SCP & $\begin{array}{c}\mathbf{A} \rightarrow \mathbf{B} \\
\& \mathbf{B} \rightarrow \mathbf{A}\end{array}$ & $\begin{array}{c}\text { Walk Forward } \\
i=2 \text { Step }=5\end{array}$ & $\begin{array}{c}\text { Step Up } \\
i=1 \text { Step }=2\end{array}$ & $\begin{array}{c}\text { Step Down } \\
i=2 \text { Step }=2\end{array}$ & $\begin{array}{c}\text { Walk Forward } \\
i=2 \text { Step }=5\end{array}$ & $\begin{array}{c}\text { Turn Right } \\
i=2 \text { Step }=4\end{array}$ & Idle & Idle & Idle & Idle \\
\hline \multirow{2}{*}{ LFP } & $\mathbf{A} \rightarrow \mathbf{B}$ & $\begin{array}{c}\text { Turn Left } \\
i=1 \text { Step }=1\end{array}$ & $\begin{array}{c}\text { Walk Forward } \\
i=2 \text { Step }=14\end{array}$ & $\begin{array}{c}\text { Turn Right } \\
i=2 \text { Step }=2\end{array}$ & $\begin{array}{c}\text { Walk Forward } \\
i=2 \text { Step }=14\end{array}$ & $\begin{array}{c}\text { Turn Right } \\
i=2 \text { Step }=3\end{array}$ & Idle & Idle & Idle & Idle \\
\hline & $\mathbf{B} \rightarrow \mathbf{A}$ & $\begin{array}{l}\text { Turn Right } \\
i=2 \text { Step }=1\end{array}$ & $\begin{array}{c}\text { Walk Forward } \\
i=2 \text { Step }=14\end{array}$ & $\begin{array}{c}\text { Turn Left } \\
i=1 \text { Step }=2\end{array}$ & $\begin{array}{c}\text { Walk Forward } \\
i=2 \text { Step }=14\end{array}$ & $\begin{array}{c}\text { Turn Left } \\
i=1 \text { Step }=3\end{array}$ & Idle & Idle & Idle & Idle \\
\hline \multirow{2}{*}{ MDP } & $\mathbf{A} \rightarrow \mathbf{B}$ & $\begin{array}{c}\text { Turn Right } \\
i=2 \text { Step }=1\end{array}$ & $\begin{array}{c}\text { Walk Forward } \\
i=2 \text { Step }=10\end{array}$ & $\begin{array}{c}\text { Turn Left } \\
i=1 \text { Step }=1\end{array}$ & $\begin{array}{c}\text { Walk Forward } \\
i=2 \text { Step }=5\end{array}$ & $\begin{array}{c}\text { Turn Left } \\
i=1 \text { Step }=1\end{array}$ & $\begin{array}{c}\text { Walk Forward } \\
i=2 \text { Step }=2\end{array}$ & $\begin{array}{c}\text { Over } i=1 \\
\text { Step }=1\end{array}$ & $\begin{array}{c}\text { Walk Forward } \\
i=2 \text { Step }=5\end{array}$ & $\begin{array}{c}\text { Turn Left } \\
i=1 \text { Step }=3\end{array}$ \\
\hline & $\mathbf{B} \rightarrow \mathbf{A}$ & $\begin{array}{c}\text { Turn Left } \\
i=1 \text { Step }=1\end{array}$ & $\begin{array}{c}\text { Walk Forward } \\
i=2 \text { Step }=5\end{array}$ & $\begin{array}{c}\text { Over } i=1 \\
\text { Step }=1\end{array}$ & $\begin{array}{c}\text { Walk Forward } \\
i=2 \text { Step }=2\end{array}$ & $\begin{array}{c}\text { Turn Right } \\
i=2 \text { Step }=1\end{array}$ & $\begin{array}{c}\text { Walk Forward } \\
i=2 \text { Step }=5\end{array}$ & $\begin{array}{l}\text { Turn Right } \\
i=2 \text { Step }=1\end{array}$ & $\begin{array}{c}\text { Walk Forward } \\
i=2 \text { Step }=10\end{array}$ & $\begin{array}{c}\text { Turn Right } \\
i=2 \text { Step }=3\end{array}$ \\
\hline
\end{tabular}


Now a function is needed to design to program the robot for performing the tasks continuously. The function is declared as Navigate( ), represented in Figure 8, which will be executed inside the robot controller continuously. This function is responsible to identify the various commands and also responsible to call the necessary subroutines depending on the lookup table as defined in the Table 1. So it is also exigency to define the various subprograms contingent on the designed lookup table. The subroutines are defined as Turn( (), ClimbUP( ), ClimbDN() and Overcome(). The subroutine for walking forward, WalkForward(), is defined in Figure 6 while designing the walking gait. All these subprograms take two parameters, Step and LR flag, with the default values as ' 0 ' to avoid the execution error if no parameter is passed to those functions. This means, if no parameter values are passed to any of these functions, the subprograms will run initializing the parameter values as ' 0 ' without causing any changes on the system current pose and position

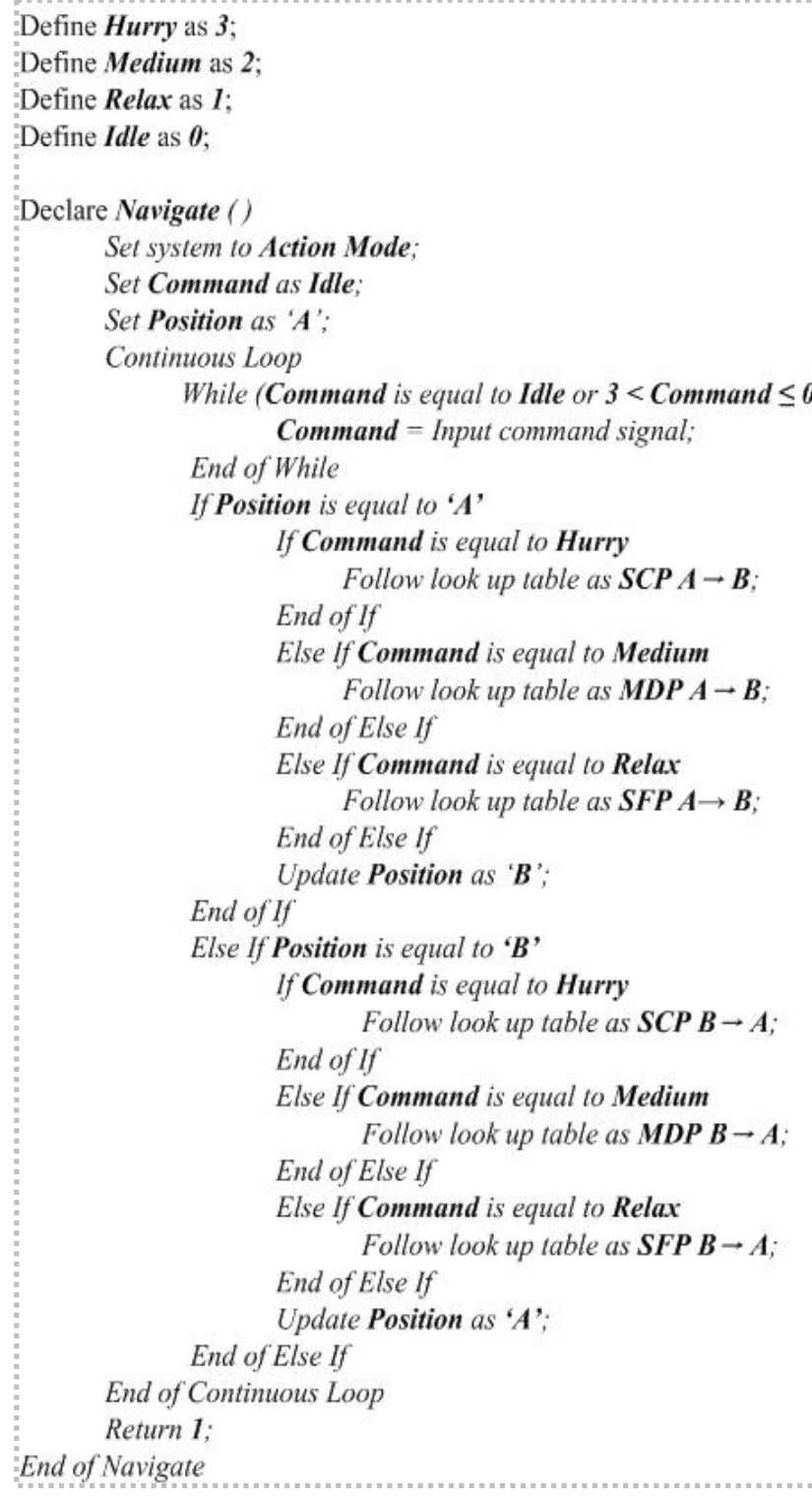

Figure 8. Definition of the Navigate() as the main continuous function

\section{Results and Observations}

The home position of the system is either A or B depicted as $H_{o m e}$ or $H_{o m e}$ based on the current idle position of the humanoid. Figure 9 is the reflections of the various attempts taken by the humanoid while navigating on its own and predefined environment. The result of the experiment presents that the system takes lowest time to navigate through the shortest path (SCP) with complex obstacle than the longest, obstacle free path (LFP). For the Medium mode, the system selects the difficult path (MDP) taking less time than the Relax mode but more than Hurry. As the validation of this concept, Equation (6) to Equation (10) are introduced to calculate the travelling times for the three different routes.

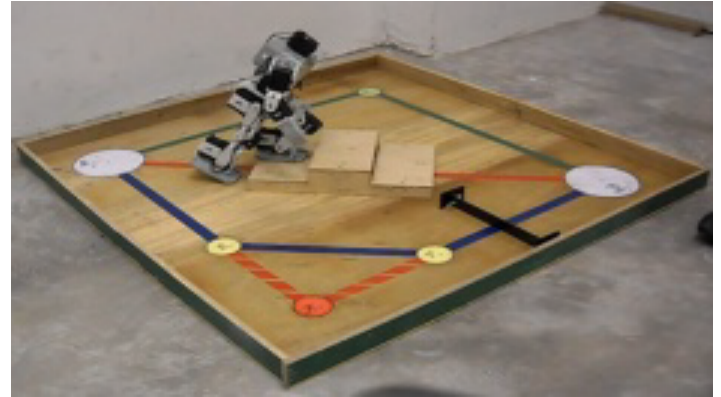

(a) Stepping up stairs on the Shortest Complex Path (SCP)

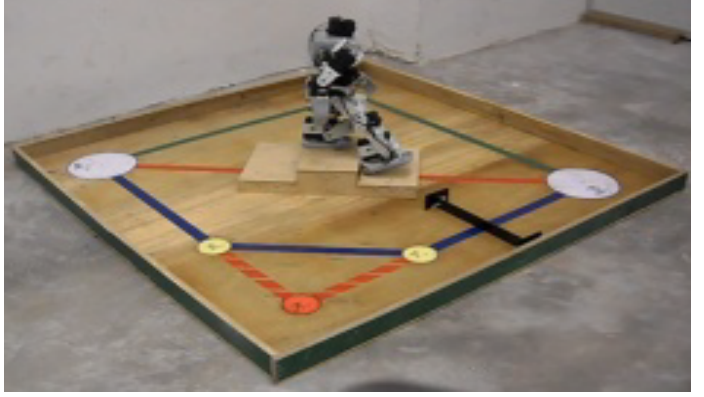

(b) Descending stairs while navigating on the SCP

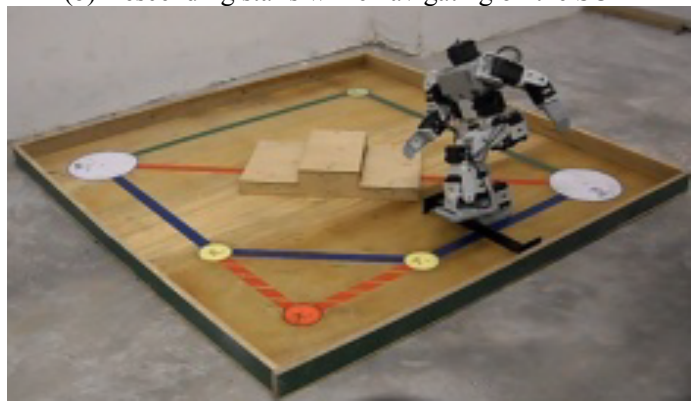

(c) Overcoming an obstacle on the Medium Difficult Path (MDP)

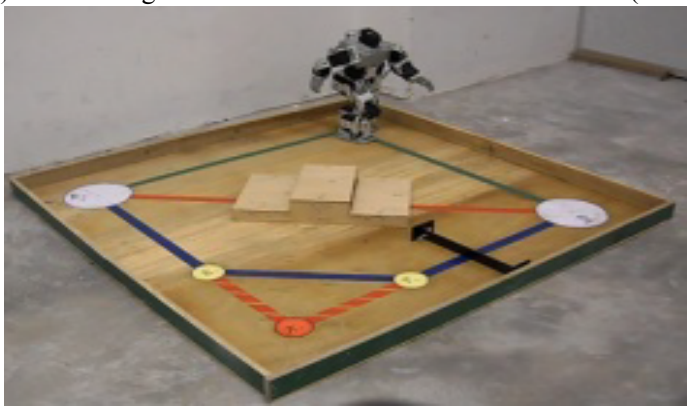

(d) Following the Longest Free Path (LFP)

Figure 9. Various actins while navigating on the designed environment 


$$
\begin{gathered}
n \text { Step }=(1 \times I S)+((n-1) \times F S)+(1 \times E S) \\
T_{n S t e p}=\left(1 \times T_{I S}\right)+\left((n-1) \times T_{F S}\right)+\left(1 \times T_{E S}\right) \\
T_{S C P(A \rightarrow B)}=T_{S C P(B \rightarrow A)} \\
=2 T_{5 \text { Step }}+2 T_{\text {StUP }}+2 T_{S t D N}+4 T_{T u S} \\
T_{M D P(A \rightarrow B)}=T_{M D P(B \rightarrow A)} \\
=6 T_{T u S}+T_{O O S}+T_{10 \text { Steps }}+2 T_{5 \text { Steps }}+T_{2 \text { Steps }} \\
T_{L F P(A \rightarrow B)}=T_{L F P(B \rightarrow A)}=6 T_{\text {TuS }}+2 T_{14 \text { Steps }}
\end{gathered}
$$

Here $I S, F S$ and $E S$ represents the Initial Step, Full Step and Ending Step respectively. $T$ stands for the time required in a particular action. The $n$ indicates the number of steps from initial to stopping action for walking. All the necessary values to calculate the required times are identified and tabulated as shown in Table 2 through the deep observation and inspection of the various gaits and the navigation strategies.

Table 2. Necessary Parameters to Calculate the Required Times

\begin{tabular}{|c|c|c|}
\hline \multirow{2}{*}{ Behavior } & \multicolumn{2}{|c|}{ Observations and Inspections } \\
\cline { 2 - 3 } & Parameters & Required Time in Second \\
\hline \multirow{3}{*}{ Walking } & $T_{I S}$ for Initial Step & $07 \mathrm{Sec}$. \\
\cline { 2 - 3 } & $T_{F S}$ for a Full Step & $08 \mathrm{Sec}$. \\
\cline { 2 - 3 } & $T_{E S}$ for Ending Step & $06 \mathrm{Sec}$. \\
\hline Turning & $T_{T u S}$ & $14 \mathrm{Sec}$. \\
\hline Obstacle Overcome & $T_{O O S}$ & $33 \mathrm{Sec}$. \\
\hline Step Up Stairs & $T_{S t U P}$ & $32 \mathrm{Sec}$. \\
\hline Step Down Stairs & $T_{S t D N}$ & $30 \mathrm{Sec}$. \\
\hline
\end{tabular}

By applying Equation (8), Equation (9) and Equation (10), the desired times that requires to navigate on the various tracks are calculated and compared with the actual times that are measured and investigated from the practical experimentation process, as demonstrated in Table 3 and Table 4. The actual error and the percent of error are also resulted in the same tables where the optimal navigation strategy is clearly visible and validated.

Table 3. Comparative Results and Validation (Test 1 with Well Charged Battery)

\begin{tabular}{|c|c|c|c|c|}
\hline \multirow{2}{*}{$\begin{array}{c}\text { Navigation } \\
\text { Intervals }\end{array}$} & \multicolumn{4}{|c|}{ Comparative Results $(\boldsymbol{T}$ SCP $<$ T_MDP $<$ T_LFP $)$} \\
\cline { 2 - 5 } & $\begin{array}{c}\text { Calculated } \\
\text { Time }\end{array}$ & Actual Time & $\begin{array}{c}\text { Error in } \\
\text { Sec. }\end{array}$ & $\begin{array}{c}\% \text { Error } \\
\text { in Sec. }\end{array}$ \\
\hline$T_{S C P}$ & $\begin{array}{c}270 \mathrm{sec} . \\
(4.30 \mathrm{~min} .)\end{array}$ & $\begin{array}{c}273 \mathrm{sec} . \\
(4.33 \mathrm{~min} .)\end{array}$ & $-3 \mathrm{sec}$. & $1.0989 \%$ \\
\hline$T_{M D P}$ & $\begin{array}{c}313 \mathrm{sec} . \\
(5.13 \mathrm{~min} .)\end{array}$ & $\begin{array}{c}317 \mathrm{sec} . \\
(5.17 \mathrm{~min} .)\end{array}$ & $-4 \mathrm{sec}$. & $1.2618 \%$ \\
\hline$T_{L F P}$ & $\begin{array}{c}318 \mathrm{sec} . \\
(5.18 \mathrm{~min} .)\end{array}$ & $\begin{array}{c}320 \mathrm{sec} . \\
(5.20 \mathrm{~min} .)\end{array}$ & $-2 \mathrm{sec}$. & $0.625 \%$ \\
\hline
\end{tabular}

Table 4. Comparative Results and Validation (Test 2 in Normal Condition)

\begin{tabular}{|c|c|c|c|c|}
\hline \multirow{2}{*}{$\begin{array}{c}\text { Navigation } \\
\text { Intervals }\end{array}$} & \multicolumn{4}{|c|}{ Comparative Results $\left(\boldsymbol{T}_{-} \boldsymbol{S C P}<\boldsymbol{T}_{-} \boldsymbol{M D P}<\boldsymbol{T} \boldsymbol{T} \boldsymbol{L F P}\right)$} \\
\cline { 2 - 5 } & $\begin{array}{c}\text { Calculated } \\
\text { Time }\end{array}$ & Actual Time & $\begin{array}{c}\text { Error in } \\
\text { Sec. }\end{array}$ & $\begin{array}{c}\% \text { Error } \\
\text { in Sec. }\end{array}$ \\
\hline$T_{S C P}$ & $\begin{array}{c}270 \mathrm{sec} . \\
(4.30 \mathrm{~min} .)\end{array}$ & $\begin{array}{c}272 \mathrm{sec} . \\
(4.32 \mathrm{~min} .)\end{array}$ & $-2 \mathrm{sec}$. & $0.7353 \%$ \\
\hline$T_{M D P}$ & $\begin{array}{c}313 \mathrm{sec} . \\
(5.13 \mathrm{~min} .)\end{array}$ & $\begin{array}{c}317 \mathrm{sec} . \\
(5.17 \mathrm{~min} .)\end{array}$ & $-4 \mathrm{sec}$. & $1.2618 \%$ \\
\hline$T_{L F P}$ & $\begin{array}{c}318 \mathrm{sec} . \\
(5.18 \mathrm{~min} .)\end{array}$ & $\begin{array}{c}320 \mathrm{sec} . \\
(5.20 \mathrm{~min} .)\end{array}$ & $-2 \mathrm{sec}$. & $0.625 \%$ \\
\hline
\end{tabular}

\section{Conclusions}

The paper draws a panoptic view of designing an algorithm for the humanoid platform to establish an optimal navigation system. The optimal navigation performance are also examined and illustrated with some comparative data analysis for various conditions of the humanoid system. Some mathematical relations and formulations are established to identify and calculate the desired time durations for the system while navigating on a particular route of the environment. The experiment is basically an inspiration and can be considered as the entrance for beginners to the vast area of this research field.

\section{ACKNOWLEDGEMENTS}

The authors would like to thank their honorable parents. They also would like to express their gratitude to the Ministry of Higher Education (MOHE), Malaysia, in funding the project through the Fundamental Research Grant Scheme (FRGS).

\section{REFERENCES}

[1] M. Akhratuzzaman and A. A. Shafie. Advancement of Android and Contribution of Various Countries in the Research and Development of the Humanoid Platform. International Journal of Robotics and Automation (IJRA), Volume (1): Issue (2), 2010.Computer Science Journal (CSC Journal), Malaysia. 43-57

[2] M. Akhtaruzzaman and A. A. Shafie. Geometrical Analysis on BIOLOID Humanoid System Standing on Single Leg. Proceedings of 2011 4th International Conference on Mechatronics (ICOM `11), 17-19 May 2011, Kuala Lumpur, Malaysia

[3] H. Qiang, K. Shuuji, K. Noriho, K. Kenji, Y. Kazuhito, A. Hirohiko, K. Kiyoshi, and T. Kazuo. A High Stability, Smooth Walking Pattern for a Biped. Robot. Proceedings of the 1999 IEEE International Conference on Robotics and Automation, Detroit, Michigan May 1999. 65-71

[4] T. Sugihara and H. Kobayashi. A Handy Humanoid Robot Navigation by Non-interruptive Switching of Guided Point and Synergetic Points. Proceedings of the 8th IEEE-RAS International Conference on Humanoid Robots, December 1 3, 2008, Daejeon, Korea

[5] C. Chevallereau, G. Bessonnet, G. Adda and Y. Aoustin. Bipedal Robots Modeling, Design and walking Synthesis. ISTE Ltd. and John Wiley \& Sons 2009

[6] M. Akhtaruzzaman, S. K. K. Hasan and A. A. Shafie. Design and Development of an Intelligent Autonomous Mobile Robot for a Soccer Game Competition. Proceedings of the 2009 International Conference on Mechanical and Electronics Engineering (ICMEE), June 27-29, 2009. Chennai, India. $167-171$

[7] S. Thrun, W. Burgard and D. Fox. A Probabilistic Approach 
to Concurrent Mapping and Localization for Mobile Robots. Autonomous Robots. Volume 5, Numbers 3-4, Kluwer Academic Publishers. Manufactured in The Netherlands. 253-271

[8] G. J. Steffen, F. Masaki and F. Masahiro. A Modular Architecture for Humanoid Robot Navigation. Proceedings of 2005 5th IEEE-RAS International Conference on Humanoid Robots, 5th Dec. 2005. 26-31

[9] S. C. Long. Ascending and Descending Stairs for a Biped Robot. IEEE Transactions On Systems, Man and Cybernetics, Part A: Systems And Humans, Vol. 29, No. 3, May 1999. $255-268$

[10] M. Stilman, K. Nishiwaki, S. Kagami and J. J. Kuffner. Planning and Executing Navigation Among Movable Obstacles. Proceedings of the 2006 IEEE/RSJ International Conference on Intelligent Robots and Systems, October 9 - 15, 2006, Beijing, China. 820-826

[11] R. Tse, A. Tay and W. Hutama. Robot Navigation using KFLANN Place Field. Proceedings of the 2008 IEEE International Conference on Systems, Man and Cybernetics (SMC 2008), 12-15 Oct. 2008, Singapore. 3034 - 3039

[12] Y. Guan, K. Yokoi, N. E. San and K. Tanie. Feasibility of Humanoid Robots Stepping over Obstacles. Proceedings of 2004 IEEWRSJ International Conference on intelligent Robots and Systems. September 28 -October 2,2004, Sendal, Japan. 130-135

[13] Y. Guan, N. E. Sian and K. Yokoi. Motion Planning for Humanoid Robots Stepping over Obstacles. IEEE/RSJ International Conference on Intelligent Robots and Systems, (IROS 2005). 2005. 363-369

[14] G. Capi, Y. Nasu, L. Barolli and K. Mitobe. Real time gait generation for autonomous humanoid robots: A case study for walking. Robotics and Autonomous Systems 42 (2003), Elsevier Science B.V. 107-116

[15] J. Chestnutt, M. Lau, G. Cheung, J. Kuffner, J. Hodgins and T. Kanade. Footstep Planning for the Honda ASIMO Humanoid. Proceedings of the 2005 IEEE International Conference on Robotics and Automation, Barcelona, Spain, April 2005. 629-634

[16] D. Katic and M. Vukobratovic. Survey of Intelligent Control Techniques for Humanoid Robots. Journal of Intelligent and Robotic Systems 37: 2003. Kluwer Academic Publishers. Printed in the Netherlands. 117-141

[17] I. W. Park, J. Y. Kim, J. Lee and J. H. Oh. Development of Humanoid Robot Platform KHR-2 (KAIST Humanoid Robot - 2). IEEE/RAS International Conference on Humanoid Robots, 2004. Vol. 1, 292-310

[18] I. W. Park, J. Y. Kim, J. Lee and J. H. Oh. Mechanical Design of Humanoid Robot Platform KHR-3(KAIST Humanoid Robot-3: HUBO). Proceedings of 2005 th IEEE/RAS International Conference on Humanoid Robots. 321-326

[19] K. Kaneko, F. Kanehiro, S. Kajita, K. Yokoyama, K. Akachi, T. Kawasaki, S. Ota and T. Isozumi. Design of Prototype Humanoid Robotics Platform for HRP. Proceedings of the 2002 IEEE/RSJ Intl. Conference on Intelligent Robots and Systems EPFL, Lausanne, Switzerland. October 2002. $2431-2436$

[20] K. Kaneko, K. Miura, F. Kanehiro, M. Morisawa, S. Nakaoka and S. Kajita. Cybernetic Human HRP-4C. Proceedings of 9th IEEE-RAS International Conference on Humanoid Ro- bots, December 7-10, 2009 Paris, France. 7-14

[21] D. Mewes and A. Heloir. The Uncanny Valley. Seminar report, DFKI and Saarland University, 2008. Retrieved on June 25, 2011. http://embots.dfki.de/doc/seminar_ss09/write up\%20uncanny\%20valley.pdf

[22] J. Carpenter, J. M. Davis, N. E. Stewart, T. R. Lee, J. D. Bransford and N. Vye. Gender Representation and Humanoid Robots Designed for Domestic Use. International Journal of Social Robots, March 19, 2009. Springer Science \& Business Media. 261-265

[23] M. Akhtaruzzaman and A. A. Shafie. Evolution of Humanoid Robots and Contribution of Various Countries in Advancing the Research and Development of the Platform. The International Conference on Control, Automation and Systems (ICCAS), Oct. 27-30, 2010 in KINTEX, Gyeonggi-do, Korea. 1021-1028

[24] M Eaton. Further Explorations in Evolutionary Humanoid Robotics. Proceedings of 12th International Symposium on Artificial Life and Robotics (ISAROB), Oita, Japan, January $25-27,2007.133-137$

[25] F. A. Siddiky, N. H. Siddique, M. O. Tokhi. A New Approach to Design and Control of Biped Robot with Minimal Number of Actuators. Proceedings of International Conference on Climbing and Walking Robots and Support Technologies for Mobile Machines 2009, 09th September 2009. 115-122

[26] A. Konno, K. Nagashima, R. Furukawa, K. Nishiwaki, T. Noda, M. Inaba and H. Inoue. Development of a Humanoid Robot Saika. Proceedings of IROS, 1997 IEEE. 805-810

[27] D. Wollherr, M. Hardt, M. Buss and O. V. Stryk. Actuator Selection and Hardware Realizationof a Small and Fast-Moving, Autonomous Humanoid Robot. Proceedings of the 2002 IEEE/RSJ International Conference on Intelligent Robots and Systems, Lausanne, Switzerland, September 30 October 4, 2002. 2491-2496

[28] E. Choong, C. M. Chew, A. N. Poo and G. S. Hong. Mechanical Design of an Anthropomorphic Bipedal Robot. First Humanoid, Nanotechnology, Information Technology, Communication and Control Environment and Management (HNICEM) International Conference, March 27-30, 2003, Manila, Philippines

[29] R. Beira, M. Lopes, M. Praca, J. S. Victor, A. Bernardino, G. Mettay, F. Becchiz and R. Saltaren. Design of the Robot-Cub (iCub) Head. Proceedings of IEEE International Conference on Robotics and Automation, Orlando, May 2006

[30] D. Gouaillier, V. Hugel and P. Blazevic. Mechatronic design of NAO humanoid. Proceedings of 2009 IEEE International Conference on Robotics and Automation Kobe International Conference Center Kobe, Japan, May 12-17, 2009. 769-774

[31] V. V. Madadi and S. Tosunoglu. Design and Development of a Biped Robot. Proceedings of the 2007 IEEE International Symposium on Computational Intelligence in Robotics and Automation. Jacksonville, FL, USA, June 20-23, 2007. 243-247

[32] Y. Matsusaka. History and Current Researches on Building a Human Interface for Humanoid Robots. Wachsmuth and G. Knoblich (Eds.): Modeling Communication, LNAI 4930, 2008. Springer-Verlag Berlin Heidelberg 2008. 109-124

[33] N. T. Phuong, D. W. Kim, H. K. Kim and S. B. Kim. An 
Optimal Control Method for Biped Robot with Stable Walking Gait. Proceedings of the 8th IEEE-RAS International Conference on Humanoid Robots, December 1-3, 2008, Daejeon, Korea. 211-218

[34] R. Simmons and S. Koenig. Probabilistic Robot Navigation in Partially Observable Environments. Proceedings of the International Joint Conference on Artificial Intelligence (IJCAI '95), July, 1995, 1080-1087

[35] M. Abdallah and A. Goswami. A Biomechanically Motivated Two-Phase Strategy for Biped Upright Balance Control. Proceedings of the 2005 IEEE International Conference on Robotics and Automation Barcelona, Spain, April 2005. 2008 $-2013$

[36] A. Goswami. Kinematic and dynamic analogies between planar biped robots and the reaction mass pendulum (RMP) model. 8th IEEE-RAS International Conference on Humanoid Robots. 1-3 Dec. 2008, Daejeon, Korea. 182 - 188

[37] C. Zhou, P. K. Yue, J. Nil and S. B. Chan. Dynamically Stable Gait Planning for a Humanoid Robot to Climb Sloping Surface. Proceedings of the 2004 IEEE Conference on Robotics, Automation and Mechatronics Singapore, 1-3 December, 2004. 341-346

[38] P. Gibbons, M. Mason, A. Vicente, G. Bugmann and P. Culverhouse. Optimization of Dynamic Gait for Bipedal Robots. Proceedings of the 2009 IEEE-RAS Intl. Conf. On Humanoid Robots (Humanoids 2009), Paris(France), 2009, December 7-10

[39] T. Takenaka. The control system for the Honda Humanoid Robot. Published by Oxford University Press on behalf of the British Geriatrics Society. Age and Ageing 2006, 35-S2: ii24-ii26

[40] H. O. Lim and A. Takanishi. Biped walking robots created at Waseda University: WL and WABIAN family, Phil. Trans. R. Soc. A (2007) 365, 49-64. Published online on 17th November 2006. Retrieved on March 25, 2011. http://rsta.royalsocietypublishing.org/content/365/1850/49.fu 11

[41] C. Schafer and R. Dillmann. Kinematic Design of a Humanoid Robot Wrist. Journal of Robotic System, 18(12). John Wiley and Sons, Inc. 747-754

[42] M. Akhtaruzzaman, A. A. Shafie and M. Rashid. Component Selection Strategy for an Anthropomorphic Robot. National Postgraduate Conference (NPC) 2011, Malaysia. 19-20 September 2011

[43] M. Akhtaruzzaman and A. A. Shafie. An Attempt to Develop a Biped Intelligent Machine BIM-UIA. Proceedings of 2011 4th International Conference on Mechatronics (ICOM '11), 17-19 May 2011, Kuala Lumpur, Malaysia

[44] M. Akhtaruzzaman and A. A. Shafie. A Novel Gait for Toddler Biped and its Control Using PIC 16F877A. Proceedings of 2011 4th International Conference on Mechatronics (ICOM `11), 17-19 May 2011, Kuala Lumpur, Malaysia

[45] M. Akhtaruzzaman, N. A. B. M. Razali, M. M. Rashid, A. A. Shafie. An Experiment on Electric Power Steering (EPS) System of a Car. Proceedings of IACSIT 2010 International Conference on Mechanical and Aerospace Engineering (ICMAE). Nov. 26 - 28, 2010. Kuala Lumpur, Malaysia. 148 $-153$
[46] M. Akhtaruzzaman, N. B. Samsuddin, N. B. Umar and M. Rahman. Design and Development of a Wall Climbing Robot and its Control System. Proceedings of 12th International Conference on Computer and Information Technology (ICCIT), December $21-23$, 2009. Independent University, Bangladesh. $309-313$

[47] M. Akhtaruzzaman, R. Akmeliawati and T. W. Yee. Modeling and Control of a Multi degree of Freedom Flexible Joint Manipulator. Proceedings of the Second International Conference on Computer and Electrical Engineering (ICCEE), December 28 - 30, 2009. Dubai, UAE. 249 - 254

[48] M. Akhtaruzzaman. Digital Bangla Clock Interfacing with Computer using Wireless media. Proceedings of the International Conference on Computer and Information Technology (ICCIT), 2005. Dhaka, Bangladesh. $401-405$

[49] M. A. Akhter. Accumulation of Research. LAP Lambert Academic Publishing, 2011

[50] S. B. Niku. Introduction to Robotics Analysis, Systems, Applications. 2001 Prentice Hall, Inc. Upper Saddle River, New Jersey 07458. 29-85

[51] L. Lee and W. E. L. Grimson. Gait Analysis for Recognition and Classification. Proceedings of the Fifth IEEE International Conference on Automatic Face and Gesture Recognition (FGR), 2002. 1-8

[52] K. G. Kim, J. Y. Lee, D. Choi, J. M. Park and B. J. You. Autonomous Task Execution of a Humanoid Robot using a Cognitive Model. Proceedings of the 2010 IEEE International Conference on Robotics and Biomimetics. December 14-18, 2010, Tianjin, China. 405-410

[53] S. Candido, Y.T. Kim and S. Hutchinson. An Improved Hierarchical Motion Planner for Humanoid Robots. Proc. IEEE-RAS Int'1. Conf. on Humanoid Robots, Daejeon, Korea, 2008, 654-661

[54] S. Candido, Y.T. Kim and S. Hutchinson. A Workspace Decomposition for Hierarchical Motion Planning with $\mathrm{Hu}-$ manoid Robots. Proc. Int'l. Conf. on Advanced Robotics, Jeju island, South Korea, 2007

[55] Y. H. Mu. Task-Oriented Architecture for a Humanoid Robot. Applied Mechanics and Materials, Vol. 40-41, Trans Tech Publications, Switzerland, 2011. 228-234

[56] S. H. Piao, X. F. Wang, W. Zhao, Q. B. Zhong. Research of Robot Gait Based on Evolution Algorithms. Journal of Applied Mechanics and Materials, Vol. 48-49, Trans Tech Publications, Switzerland, 2011. 928-931

[57] M. Akhtaruzzaman, N. A. B. M. Razali, M.. M. Rashid, A. A. Shafie. An Experiment on Electric Power Steering (EPS) System of a Car. Journal of Applied Mechanics and Materials Vols. 110-116, Trans Tech Publications, Switzerland, 2012. $4941-4950$

[58] X. F. Wang, B. R. Hong, Q. B. Zhong, S. H. Piao. A New Robot Stance Control Method. Journal of Advanced Materials Research, Vols. 255-260, Trans Tech Publications, Switzerland, 2011. 2101-2105

[59] Q. J. Du, L. P. Li, B. Dai. Object Manipulation Control Strategy Analysis Center of Humanoid Robot. Journal of Advanced Materials Research, Vols. 267, Trans Tech Publications, Switzerland, 2011. 904-908 
[60] Q. J. Du, C. Sun, X. G. Huang. Motion Control System Design of a Humanoid Robot Based on Stereo Vision. Journal of Applied Mechanics and Materials, Vols. 55-57, Trans Tech Publications, Switzerland, 2011. 877-880

[61] T. J. Zhao. Study Situation and Key Technology of Mobile Humanoid Robot. Journal of Advanced Materials Research Vols. 295-297, Trans Tech Publications, Switzerland, 2011. 450-453

[62] T. J. Zhao. Dynamic Cooperative Manipulating Pattern Generation for Mobile Humanoid Robot Using Waist Moment Compensation. Journal of Advanced Materials Research Vols. 201-203, Trans Tech Publications, Switzerland, 2011. 1978-1982

[63] Q. J. Du, X. Y. Zhang, X. G. Huang. Modeling and Analysis of a Humanoid Robot Active Stereo Vision Platform. Journal of Applied Mechanics and Materials, Vols. 55-57, Trans Tech Publications, Switzerland, 2011. 868-871

[64] T. Li, B. Hu. Research and Application of Intelligent Humanoid Robot. Journal of Applied Mechanics and Materials, Vols. 44-47, Trans Tech Publications, Switzerland, 2011. 456-460

[65] G. C. Yu, Z. L. Wang, L. Xie, J. M. Xu. Network-Based Humanoid Robot Remote Interaction with the Actual Situation Fusion Technology. Journal of Advanced Materials Research Vols. 271-273, Trans Tech Publications, Switzerland, 2011. 191-196

[66] T. Y. Wu, T. J. Yeh, B. H. Hsu. Trajectory Planning of a One-Legged Robot Performing a Stable Hop. The International Journal of Robotics Research (IJRR), 19 Jan. 2011 $01-20$

[67] J. W. Hurst. The Electric Cable Differential Leg: A Novel Design Approach for Walking and Running. International Journal of Humanoid Robotics (IJHR), Vol. 8, Issue: 2 (2011), 301-321
[68] J. Liu, F. Xue and X. Chen. A Universal Biped Walking Generator for Complex Environments with Pattern Feasibility Checking. International Journal of Humanoid Robotics (IJHR), Vol. 8, Issue: 2 (2011), 323-357

[69] M. Tarokh and M. Wang. A Computational Intelligent Approach to Kinematics Problems in Robotics and Computer Animation. International Journal of Humanoid Robotics (IJHR), Vol. 8, Issue: 2 (2011), 375-390

[70] F. A. Siddiky, M. S. Alam and T. Ahsan. High Performance Reliable Obstacle Detection and Height Measurement by Stereo Camera for Intelligent Home Service Robot. 10th International Conference on Computer and Information Technology (ICCIT), 27-29 Dec. 2007, Dhaka, Bangladesh. 01-06

[71] R. Challoo, P. Rao, S. Ozcelik, L. Challoo, and S. Li. Navigation Control and Path Mapping of a Mobile Robot using Artificial Immune Systems. International Journal of Robotics and Automation, Vol. (1), Issue (1), 2010. 01-25

[72] A. Iqbal, A. A Shafie, M. R. Khan, M. F. Alias and J. Radhi. HRI for Interactive Humanoid Head Amir-II for Visual Tracking and Servoing of Human Face. International Journal of Robotics and Automation (IJRA), Vol. (2), Issue (3), 2011. 220-231

[73] S. Mondal, A. Nandy, Chandrapal, P. Chakraborty and G. C. Nandi. A Central Pattern Generator based Nonlinear Controller to Simulate Biped Locomotion with a Stable Human Gait Oscillation. International Journal of Robotics and $\mathrm{Au}-$ tomation (IJRA), Vol. (2), Issue (2), 2011. 93-106

[74] S. Garrido, L. Moreno, D. Blanco and P. Jurewicz. Path Planning for Mobile Robot Navigation using Voronoi Diagram and Fast Marching. International Journal of Robotics and Automation (IJRA), Vol. (2), Issue (1), 2011. 42-64

[75] Y. Liu. A Robotic Prototype System for Child Monitoring. International Journal of Robotics and Automation (IJRA), Vol. (2), Issue (1), 2011. 65-77 